\title{
Functional Parcellation of the Lateral Mesencephalus
}

\author{
Carine Karachi, ${ }^{1,2 *}$ Arthur André, ${ }^{1 *}$ Eric Bertasi, ${ }^{3}$ Eric Bardinet, ${ }^{3}$ Stéphane Lehéricy, ${ }^{3}$ and Frédéric A. Bernard ${ }^{4}$ \\ ${ }^{1}$ Assistance Publique-Hôpitaux de Paris, Groupe Hospitalier Pitié-Salpêtrière, 75013 Paris, France, ${ }^{2}$ Université Pierre et Marie Curie-Paris 6, CR-ICM, UMR- \\ S975, 75013 Paris, France, ${ }^{3}$ Centre de NeuroImagerie de Recherche, Centre de Recherche de l'Institut du Cerveau et de la Moelle épinière, Université Pierre \\ et Marie Curie-Paris 6 UMR-S975, Inserm U975, CNRS UMR7225, Groupe Hospitalier Pitié-Salpêtrière, 75013 Paris, France, and ${ }^{4}$ Laboratoire d'Imagerie et \\ de Neurosciences Cognitives, UMR 7237, CNRS/Université de Strasbourg, 67000 Strasbourg, France
}

The mesencephalic locomotor region (MLR), which includes the pedunculopontine nucleus (PPN) and the cuneiform nucleus (CN), has been recently identified as a key structure for locomotion and gait control in mammals. However, the function and the precise anatomy of the MLR remain unclear in humans. To study the lateral mesencephalus, we used fMRI in 15 right-handed healthy volunteers performing two tasks: imagine walking in a hallway and imagine an object moving along the same hallway. Both tasks were performed at two different speeds: normal and 30\% faster. We identified two distinct networks of cortical activation: one involving motor/premotor cortices and the cerebellum for the walking task and the other involving posterior parietal and dorsolateral prefrontal cortices for the object moving task. In the lateral mesencephalus, we found that two different but anatomically connected parts of the MLR were activated during the fast condition of each task. The CN and the dorsal part of the PPN were activated during the fast imaginary walking task, whereas the ventral part of the PPN and the ventral part of the reticular formation were activated while subjects were imagining the object moving fast. Our data suggest that the lateral mesencephalus participates in different aspects of gait in humans, with the $\mathrm{CN}$ and dorsal PPN controlling motor aspects of locomotion and the ventral PPN being involved in integrating sensory information.

\section{Introduction}

The mesencephalic locomotor region (MLR), which includes the pedunculopontine nucleus (PPN) and the cuneiform nucleus $(\mathrm{CN})$, is important for gait in mammals. The MLR receives inputs from the cortex, the basal ganglia, and the cerebellum, and projects to ascending thalamocortical and descending reticulospinal pathways. This unique anatomical position suggests that the MLR integrates diverse information to produce locomotion (Takakusaki, 2008; Alam et al., 2011).

The PPN and CN were identified in humans (Olszewski and Baxter, 1954) as groups of cholinergic, glutamatergic, and GABAergic neurons located in the reticular formation. The cholinergic neurons serve to delineate PPN boundaries, identifying a pars compacta (richer in cholinergic neurons) and a pars dissipata (Mesulam et al., 1989). The CN lies dorsal to the PPN and ventral to the colliculi but both nuclei are difficult to delineate because of a lack of clear boundaries. Functionally, lesions of the anterior MLR in animals induce motor disabilities (Jenkinson et

\footnotetext{
Received Feb. 2, 2012; revised April 13, 2012; accepted April 17, 2012

Author contributions: C.K., E. Bardinet, S.L., and F.A.B. designed research; A.A. and E. Bertasi performed research; E. Bardinet contributed unpublished reagents/analytic tools; E. Bardinet and F.A.B. analyzed data; C.K. and A.A. wrote the paper.

This research was supported by a grant from the Michael J Fox Foundation. We thank the medical students who participated as healthy volunteers. We thank Drs. Chantal François and Jérôme Yelnik for their anatomical expertise and for comments on an earlier version of this manuscript. Lydia Yahia-Cheriff provided helpful comments concerning statistical analysis of interactions. We are grateful to Dr. Brian Lau for carefully revising this manuscript.

${ }^{*}$ C.K. and A.A. contributed equally to this work.

The authors declare no financial conflicts of interest.

Correspondence should be addressed to Carine Karachi, Assistance Publique-Hôpitaux de Paris, Groupe Hospitalier Pitié-Salpêtrière, 47 boulevard de I'Hôpital, 75013 Paris, France. E-mail: carine.karachi@gmail.com.

DOI:10.1523/JNEUROSCI.0509-12.2012

Copyright $\odot 2012$ the authors $\quad 0270-6474 / 12 / 329396-06 \$ 15.00 / 0$
}

al., 2009) whereas lesions of the posterior MLR induce cognitive dysfunction (Alderson et al., 2008). More specifically, a PPN cholinergic lesion is sufficient to induce gait deficits in monkeys ( $\mathrm{Ka}$ rachi et al., 2010). Moreover, patients with Parkinson's disease (PD) including cholinergic cell loss show more severe motor disabilities with gait disorders (Rinne et al., 2008; Bohnen et al., 2009). Recently, PPN stimulation has been proposed to treat freezing of gait in PD patients with the aim of stimulating remaining neurons. Despite promising initial results (Mazzone et al., 2008), further clinical studies showed only mild alleviation (Ferraye et al., 2010; Moro et al., 2010), suggesting that a better understanding of the MLR is necessary (Mazzone et al., 2011). Therefore, we studied the MLR in normal subjects using fMRI, seeking a functional parcellation that could provide a better delineation of the areas involved in the motor aspects of gait control.

We used a validated task of imagery of gait (Bakker et al., 2008) and adapted it (Normal and Fast conditions). Imagination of gait is well known to reveal a motor/premotor network in fMRI (Iseki et al., 2008). Activations are also found in the midbrain tegmentum and the pontomesencephalic region (Jahn et al., 2004; la Fougère et al., 2010). More precisely, mesencephalic activations are observed during the imagination of running (Jahn et al., 2008) and the imagination of gait in PD patients with freezing (Snijders et al., 2011). Moreover, it has been shown that increasing stepping frequency increases the activity of PPN neurons recorded in PD patients undergoing PPN deep brain stimulation (Piallat et al., 2009). Initiation and velocity seem to be important gait parameters, which enhance activation in the MLR. Since the MLR is also involved in cognitive functions (Stefani et al., 2010), we added a control task consisting of imagining an object moving 
with the same spatiotemporal context. Knowing that in animals, the anterior MLR is related to motor control and the posterior MLR is involved in cognitive functions, we hypothesized that we could obtain a similar parcellation in humans. Some of the results of this study were previously reported in Karachi et al. (2010).

\section{Materials and Methods}

Subjects. Fifteen healthy volunteers (mean age: $24.7 \pm 1.3$ years; 7 females, 8 males) participated in the fMRI experiment. The local ethics committee approved the study, and we obtained written informed consent from all subjects in accordance with the Declaration of Helsinki. All participants were consistent right-handers (Edinburgh Handedness Inventory mean score: $89.88 \pm 2.91 \%$ ) (Oldfield, 1971).

Experimental tasks. We used a modified version of a previously validated paradigm of imaginary gait and object movement (Bakker et al., 2008). Subjects were trained to perform both tasks at two different speeds (normal and 30\% faster) as described previously (Karachi et al., 2010). After training, subjects first saw a written instruction on a screen in the MRI scanner [Normal IG (imagery of gait), Faster IG, Normal IOM (imagery of object moving), Fast IOM]. Subjects were then instructed to close their eyes and press a button to signal that they had started to imagine the required task. Stimulus presentation was controlled using a $\mathrm{PC}$ running Cogent software. Motor responses (button presses with the right thumb) were recorded via an MRI-compatible one-button stick (Current Designs). The IG and IOM trials ( 80 trials, 20 per condition) were performed in two runs of $\sim 15$ min each. For each run, the trial order was pseudorandomized across the experimental factors (i.e., IG or IOM and normal or faster speed).

Data acquisition. Imaging data were collected at the Centre for NeuroImaging Research of Pitié-Salpêtrière Hospital using a 3 tesla Siemens Trio scanner. T2*-weighted echo-planar images depicting BOLD contrast were acquired in a single session (echo time, $30 \mathrm{~ms}$; repetition time, $2.8 \mathrm{~s} ; 40$ coronal slices, interleaved acquisition; voxel size, $1.6 \times 1.6 \times 1.6$ $\mathrm{mm}^{3}$; field of view, $204.8 \mathrm{~mm}^{2}$ ). Positioning and orientation of this field of view (FOV) was carefully set up to include both the MLR and the sensorimotor cortex including the supplementary motor area (SMA). This was done by orienting, on a sagittal view, the FOV parallel to the floor of the fourth ventricle. High-resolution anatomical images were acquired using an MPRAGE sequence (echo time, $4.18 \mathrm{~ms}$; repetition time, $2.3 \mathrm{~s}$; 176 sagittal slices; voxel size, $1.0 \times 1.0 \times 1.0 \mathrm{~mm}^{3}$; field of view, $256 \mathrm{~mm}^{2}$ ).

Data preprocessing. Imaging data were preprocessed with SPM5 statistical parametric mapping software (http://www.fil.ion.ucl.ac.uk/spm) implemented on MATLAB (MathWorks). Images were corrected for differences in timing of slice acquisition, followed by rigid body motion correction. The motion parameters for translation $(x, y, z)$ and rotation (yaw, pitch, roll) were included as covariates of noninterest in the general linear model. Following the coregistration of functional and structural images, the anatomical images were segmented and normalized to stereotactic Montreal Neurological Institute (MNI) space. Functional images were then normalized using the same normalization parameters and smoothed with a $10 \mathrm{~mm}$ full-width at half-maximum isotropic Gaussian kernel. The time series were high-pass filtered (to a maximum of 1/128 $\mathrm{Hz}$ ) to remove low-frequency noise.

Data analysis. The four event types of the $2 \times 2$ factorial experimental designs were modeled: Normal IG, Fast IG, Normal IOM, and Fast IOM. The average hemodynamic response to each event type was modeled using a canonical, synthetic hemodynamic response function (Friston et al., 1998). Events were treated as delta-functions (duration $=0$ ) and onsets were aligned to the button press marking the onset of imagery. Effects of interest were examined in each subject using linear contrasts. The resulting subject-specific contrast images were then entered into second-level random-effects analyses using one-sample $t$ tests. Since we had strong a priori hypotheses regarding the involvement of the MLR during imaginary gait, a threshold of $p<0.001$ (uncorrected for multiple comparisons; cluster size $>30$ voxels) was chosen.
Table 1. Brain regions showing significant signal increases for the following comparisons

\begin{tabular}{|c|c|c|c|c|c|}
\hline \multirow[b]{2}{*}{ Brain region } & \multirow[b]{2}{*}{ BA } & \multicolumn{3}{|c|}{ MNI peak coordinates } & \multirow[b]{2}{*}{$t$ value } \\
\hline & & $x$ & $y$ & $z$ & \\
\hline \multicolumn{6}{|l|}{ Fast IG versus Normal IG } \\
\hline L. MLR & & -5 & -26 & -10 & 4.77 \\
\hline L. Cerebellum & & 21 & -59 & -24 & 4.04 \\
\hline R. Cerebellum & & 24 & -50 & -43 & 3.78 \\
\hline L. Cerebellum & & -34 & -48 & -30 & 3.38 \\
\hline \multicolumn{6}{|l|}{ Fast IOM versus Normal IOM } \\
\hline L. Hippocampus & & -29 & -26 & -5 & 3.90 \\
\hline L. MLR & & -8 & -21 & -16 & $2.96^{*}$ \\
\hline L. MLR & & -2 & -27 & -16 & $2.89^{*}$ \\
\hline \multicolumn{6}{|l|}{ IG versus IOM } \\
\hline R. Cerebellum & & 5 & -46 & -43 & 4.68 \\
\hline R. Precentral gyrus & 6 & 16 & -16 & 72 & 4.64 \\
\hline L. Precentral gyrus & 6 & -11 & -13 & 70 & 3.97 \\
\hline L. Posterior cingulate & 23 & -16 & -37 & 30 & 4.16 \\
\hline L. Cerebellum & -19 & -38 & -27 & 4.14 & \\
\hline R. Superior temporal gyrus & 22 & 48 & -29 & 0 & 3.93 \\
\hline R. Precuneus & 19 & 14 & -53 & 16 & 3.74 \\
\hline L. Precuneus & 19 & -18 & -46 & 6 & 3.72 \\
\hline L. Middle frontal gyrus & 8 & -35 & 11 & 59 & 3.58 \\
\hline L. Parahippocampal gyrus & 37 & -24 & -40 & -8 & 3.58 \\
\hline L. Thalamus & & -10 & -19 & 21 & 3.57 \\
\hline R. Cerebellum & & 22 & -34 & -27 & 3.48 \\
\hline \multicolumn{6}{|l|}{ IOM versus IG } \\
\hline R. Precentral gyrus & 6 & 61 & 8 & 18 & 5.85 \\
\hline L. Supramarginal gyrus & $40 / 2$ & -59 & -29 & 40 & 5.38 \\
\hline L. Precentral gyrus & $6 / 9$ & -58 & 5 & 30 & 4.26 \\
\hline L. rolandic operculum & 44 & -40 & -6 & 11 & 4.05 \\
\hline R. Supramarginal gyrus & 40 & 48 & -34 & 45 & 3.90 \\
\hline
\end{tabular}

Brain regions showing significant signal increases for the following comparisons: Fast IG versus Normal IG; Fast IOM versus Normal IOM; IG versus IOM; IOM versus IG. The results of the first and third contrasts were previously published in Karachi et al. (2010). BA, Brodmann area; L., left; R., right. ${ }^{*} p<0.005$.

\section{Results}

\section{Behavioral data}

There were no significant differences in the imagery times between the IG and IOM tasks $\left(F_{(1,11)}=0.011, p=0.92\right)$. In both tasks, the faster speed resulted in shorter imagery times $\left(F_{(1,11)}=\right.$ $9.5, p=0.003)$. There was no significant task-by-speed interaction $\left(F_{(1,11)}=0.016, p=0.899\right)$.

\section{Functional MRI data}

\section{Cortical activations}

Imagery of gait: IG versus IOM. The comparison between the IG and IOM conditions (IG vs IOM) yielded significant activations, mainly in a network of motor-specific brain regions. These clusters of activation included bilateral premotor cortex in the precentral gyrus, the SMA in the frontal part of the paracentral lobule (Brodmann area 6), the anterior lobes of the cerebellum bilaterally, the left parahippocampal gyrus (Brodmann area 37), the left posterior cingular sulcus (Brodmann areas 23), the right superior temporal gyrus (Brodmann area 22), the right and left precuneus (associative visual cortex, Brodmann Area 19), the superior frontal gyrus (frontal eye field, Brodmann area 8), and the left thalamus. Results are given in MNI coordinates and corresponding approximate Brodmann areas in Table 1 and cortical activations are shown in Figure $1 A$ in yellow.

Imagery of gait: Fast IG versus Normal IG. The comparison between faster and normal IG (Fast IG vs Normal IG) yielded a significant bilateral activation in the cerebellum.

Imagery of object movement: IOM versus IG. The comparison between the IOM and IG conditions (IOM vs IG) revealed significant cortical activations in bilateral parietal cortices, with a left 

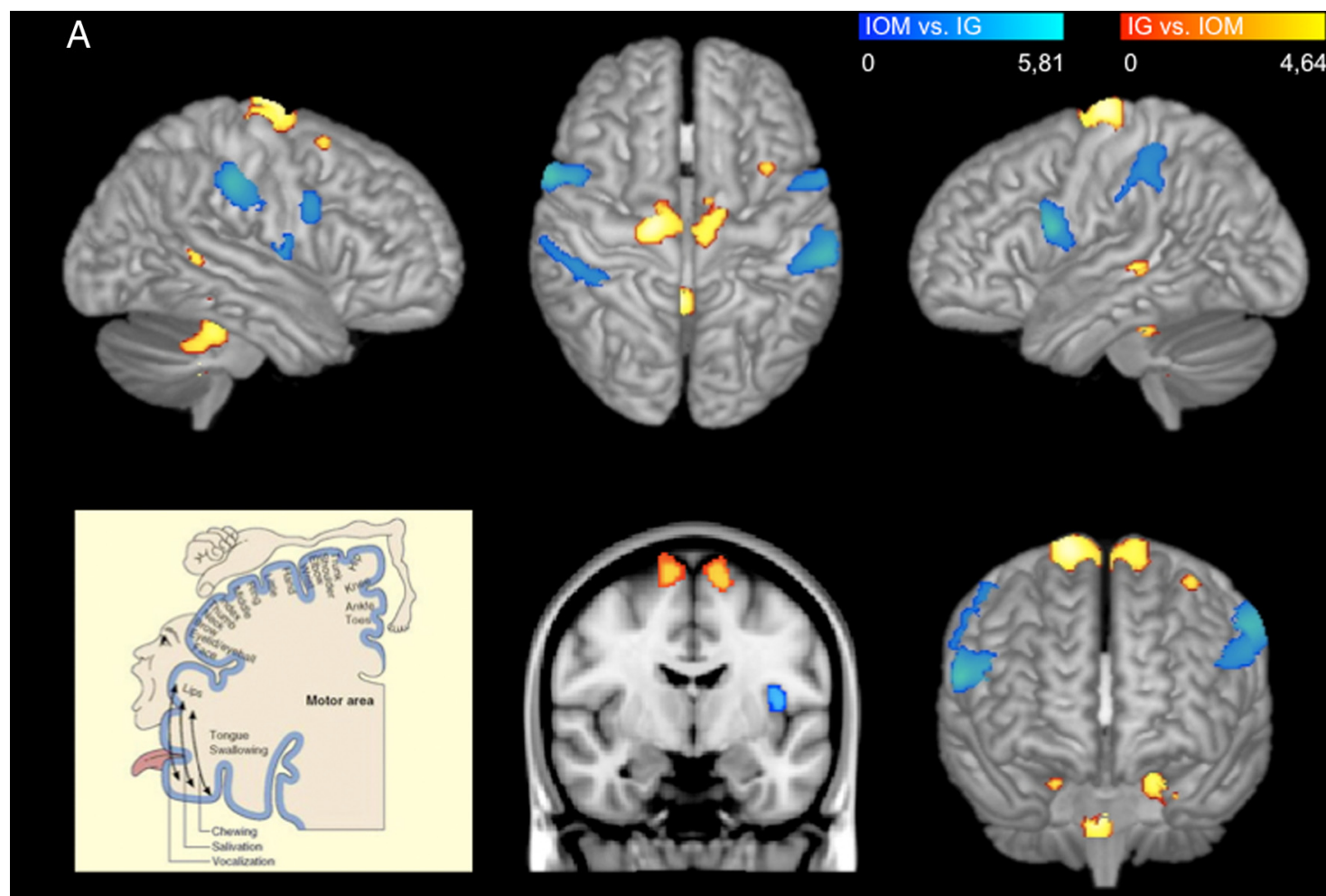

B
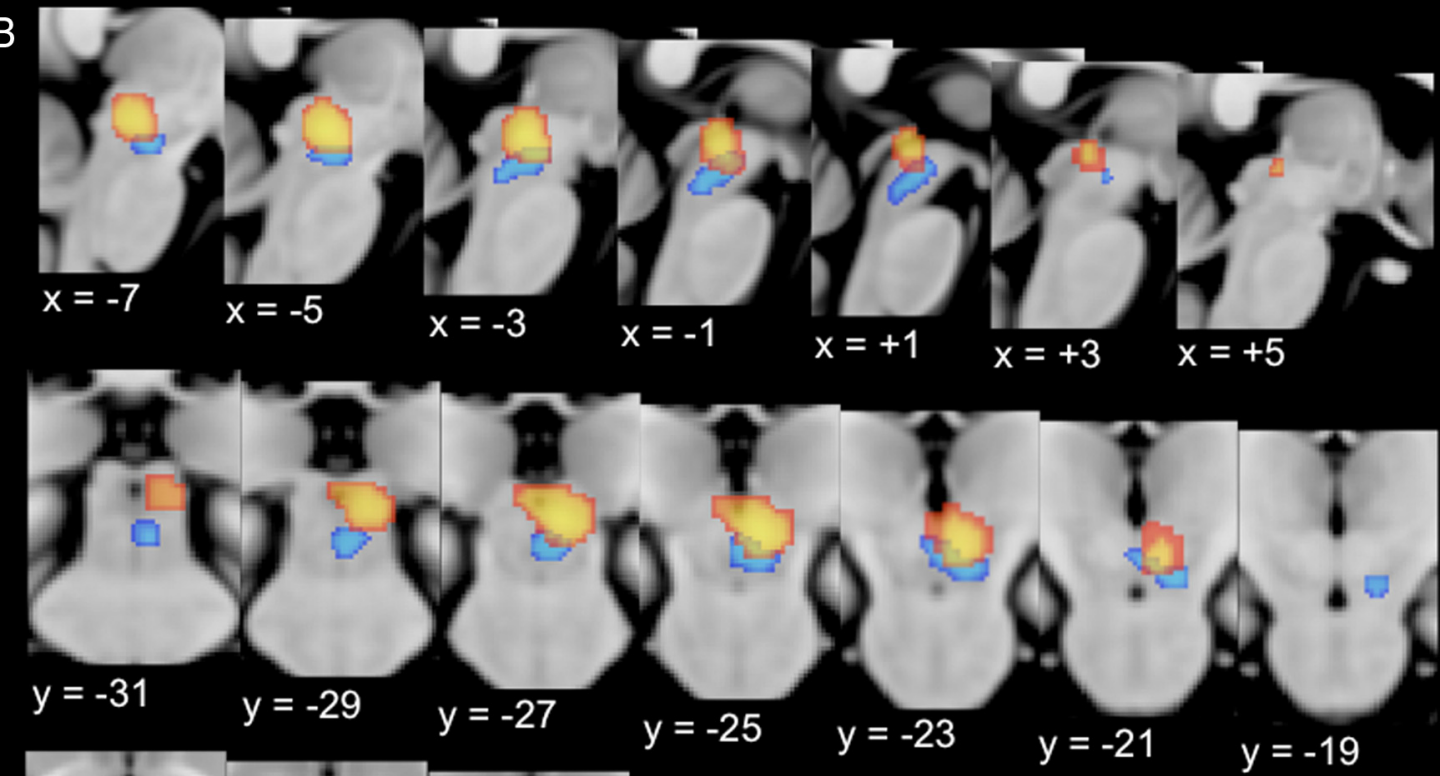


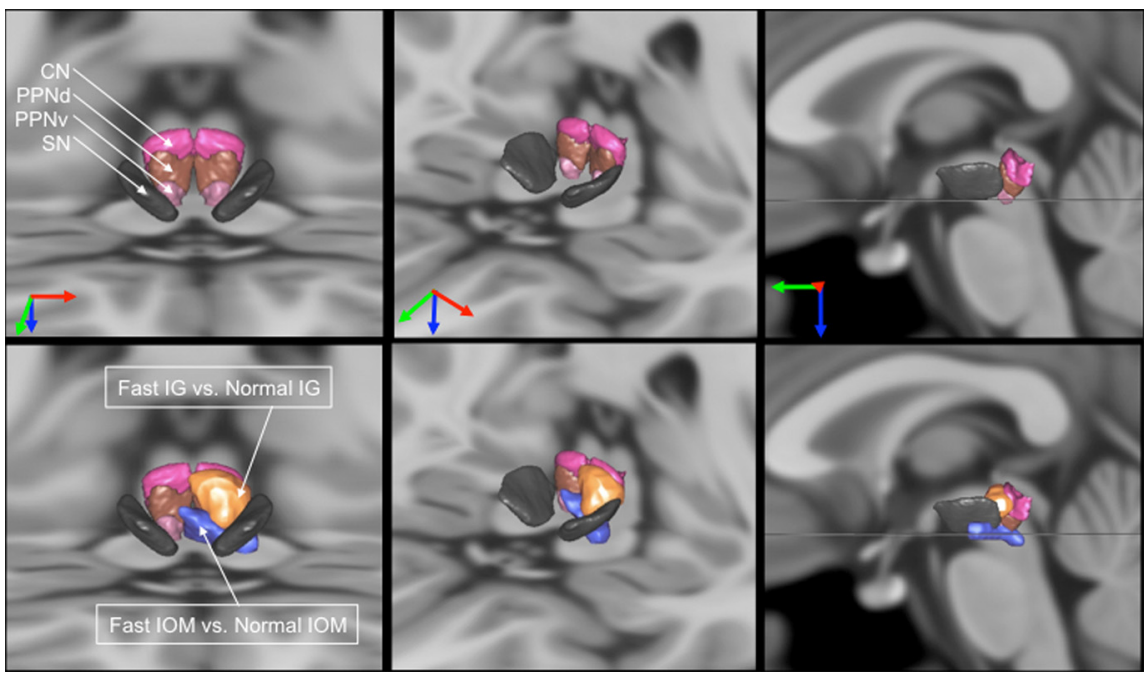

Figure 2. MLR activations with respect to MLR structures in the basal ganglia atlas. Illustration of the MLR activations with respect to MLR nuclei [CN, dorsal PPN (PPNd), ventral PPN (PPNv), parts of the pedoculopontine nucleus) and the substantia nigra (SN). To better understand the overlap between the activations and the nuclei, we show three viewpoints of the nuclei (top) and the same viewpoints with the activations together with the nuclei (bottom). The orientation of the viewpoints is indicated by the arrows (red, right to left; green, back to front; blue, top to down). In the right column, the viewpoint is strictly sagittal, the horizontal line representing the transverse MRI section visible on the two other viewpoints at the level of the ventral PPN. The nuclei come from the 3D histological and deformable YeB atlas (Yelnik et al., 2007; Bardinet et al., 2009). This atlas has been mapped onto the MNI152 template through a validated intensity-based deformation procedure that is adapted to subcortical structures.

a significant activation in the left MLR, suggesting that this region might be activated during IOM but at a lower level. Indeed, when using a less stringent threshold ( $p<0.005$, uncorrected), the comparison between fast and normal IOM (Fast IOM vs Normal IOM) revealed a specific activation in the ventral part of the PPN, which extends more ventrally into the reticular formation (Table 1; Fig. $1 B$; Fig. 2, blue). This effect was also significant by a more controlled regional analysis of voxels in a $8 \mathrm{~mm}$ radius centered on left MLR $[x=-8, y=-21, z=$ -16 ; coordinates obtained from the contrast (Fast IOM vs Normal IOM)], using a small volume correction to maintain family-wise error at $5 \%$.

\section{Task-by-speed interaction}

The whole-brain analysis did not highlight any significant (at the threshold of $p<0.001$ ) task (IG, IOM) by speed (Normal, Faster) interaction effects because the individual significant clusters in the midbrain are small and do overlap. However, to avoid the possibility to miss a relevant interaction effect, we extracted

dominance, including the left lateral intraparietal gyrus in the posterior parietal cortex (supramarginal gyrus, Brodmann area 40), a part of left somatosensory area (Brodmann area 2) and right supramarginal gyrus (Brodmann area 40), a cluster of activation in the dorsolateral prefrontal cortex and a part of premotor area (Brodmann areas 6 and 9), and the rolandic operculum (Brodmann area 44). Results are given in MNI coordinates and corresponding approximate Brodmann areas in Table 1 and cortical activations are shown in Figure $1 A$ in blue.

Imagery of object movement: Fast IOM versus Normal IOM. The comparison between fast and normal IOM (Fast IOM vs Normal IOM) yielded a significant activation in the left hippocampus.

\section{Midbrain activations}

Imagery of gait: IG versus IOM. The comparison between the IG and IOM conditions (IG vs IOM) did not reveal any significant activation in the midbrain.

Imagery of gait: Fast IG versus Normal IG. The comparison between fast and normal IG (Fast IG vs Normal IG) yielded a significant activation in the left MLR. This cluster of activation includes both the $\mathrm{CN}$ and the dorsal part of PPN (Table 1; Fig. $1 B$; Fig. 2, yellow). This effect was also significant using a more controlled regional analysis of voxels in a $8 \mathrm{~mm}$ radius centered on left MLR $[x=-5, y=-26, z=-10$; coordinates obtained from the contrast (Fast IG vs Normal IG)], using a small volume correction to maintain family-wise error at $5 \%$.

Imagery of object movement: IOM versus IG. The comparison between the IOM and IG conditions (IOM vs IG) did not reveal any significant activation in the midbrain.

Imagery of object movement: Fast IOM versus Normal IOM. At the preset threshold ( $p<0.001$, uncorrected), the comparison between fast and normal IOM (Fast IOM vs Normal IOM) did not reveal any significant activation within the midbrain. However, a direct comparison between the IG and IOM tasks [(Fast IG vs Normal IG) - (Fast IOM vs Normal IOM)] also did not yield the $\mathrm{fMRI}$ activity in two specific and nonoverlapping areas of the $\operatorname{MLR}(x=-5, y=-26, z=-10 ; x=-8, y=-21, z=-16)$ at the locations of the peak activation for the contrasts (Fast IG vs Normal IG) and (Fast IOM vs Normal IOM) (Table 1), respectively. For each of these two areas, we performed a two-by-two ANOVA with task (IG, IOM) and speed (Normal, Fast) as factors, which revealed a significant task-by-speed interaction effect in the first $(F=8.49 ; p=0.0113)$ but not in the second $(F=0.51$; $p=0.4874)$ area. These results confirm the specific functional involvement of each MLR cluster in both tasks.

\section{Anatomical location of activations in the lateral mesencephalus}

Figure 2 shows the MRI parcellation of the human lateral mesencephalus with $\mathrm{CN}$ and PPN superimposed using a deformable 3D anatomical atlas (Yelnik et al., 2007; Bardinet et al., 2009).

\section{Discussion}

We identified a gait network using fMRI in healthy volunteers performing a validated imagery of gait task. This gait network includes several motor regions such as motor and premotor cortices and SMA. The bilateral activation in the motor cortex involved the area devoted to the lower limbs representation of Penfield (1954), confirming that subjects performed the task. These data are in line with prior work demonstrating that imagery of gait is a good tool to study real locomotion (Wang et al., 2009), even if some differences exist (Fink et al., 1997; la Fougère et al., 2010). As illustrated on Figure $1 A$, the upper activations associated with the imagery of gait task are located, compared with the Penfield's (1954) homunculus, in motor cortical areas corresponding to the pelvic belt and upper muscles of the lower limbs, which are involved in the initiation and the continuing execution of gait. We also found a significant activation in the cerebellum, which is well known for controlling posture and locomotion in humans and animals. As shown in decerebrate cats, 
microstimulation of the vermis increase the postural muscle tone (Mori et al., 2000). Also part of this network, we found activations in the cingulate cortex, which has been previously associated with the mental representation of motor action (Jeannerod and Decety, 1995).

At the brainstem level, we found a significant activation in the MLR, which was only apparent when subjects performed the fast condition of the IG task. This activation was localized in the left $\mathrm{CN}$ and dorsal PPN. This unilateral activation suggests a dominance of left mesencephalic area for gait control in our righthanded subjects. This left dominance may be due to the possibility that some higher-order motor functions are more controlled by the left hemisphere for both left- and right-limb movements (Haaland et al., 2000; Janssen et al., 2011), but we cannot exclude that it is due to the global dominance of the left side of the brain in our right-handed subjects. This $\mathrm{CN}$ and dorsal PPN activation suggests that this specific MLR area is involved when the velocity of gait changes. This speed effect was already noted in previous fMRI studies in which large mesencephalic activations were observed while healthy volunteers were imagining themselves running (Jahn et al., 2008).

It is worth considering the fact that, as we modeled the imagery trials with a delta-function (peak at $6 \mathrm{~s}$ after onset), there might be a difference between the fastest trials, for which the different steps of the trials (initiation, acceleration, and termination) would be all modeled, and the longest trials, for which the termination step would not be systematically modeled. We cannot exclude an effect of this difference on the activity in MLR. In the fast condition of IG, the acceleration is also greater than in the normal IG. The MLR activation could also be related to this change in the initiation of the gait program rather than the velocity itself. This would be in line with the idea that changing and/or adapting gait could activate the MLR and not the automatic gait program itself (la Fougère et al., 2010). This interpretation could also explain why a similar activation has been found in patients with freezing of gait (Snijders et al., 2011), a model of a total lack of initiation of the gait program.

The $\mathrm{CN}$ and the dorsal part of the PPN appear to be part of the gait motor/premotor network. This idea is motivated by early observations that electrical stimulation in the MLR of cats can drive the transition of stepping frequency from a walk to a gallop (Shik et al., 1966; Grillner, 1981; Mori et al., 1989). More specifically, stimulating the $\mathrm{CN}$ has been shown to produce locomotion whereas stimulating the PPN produced change in muscular tone (Jordan et al., 2008; Takakusaki, 2008). In addition, electrophysiological recording during deep brain stimulation (DBS) in PD patients have shown that individual dorsal PPN neurons increase their firing rates with increased stepping frequency (Piallat et al., 2009). A direct monosynaptic pathway linking CN/PPN and motor cortices has been demonstrated in monkeys using tract tracing and diffusion tensor imaging (Aravamuthan et al., 2007, 2009; Muthusamy et al., 2007). Our finding supports the idea that this part of the MLR controls motor aspects of gait in humans and could help to target the lateral mesencephalus for DBS in PD patients with gait disorders. Indeed, the PPN has been recently proposed as a target for deep brain stimulation to alleviate gait disorders in PD patients. The first studies using DBS to treat advanced PD patients concluded that low-frequency stimulation of the PPN could be effective to control freezing of gait and falls (Mazzone et al., 2005), although the precise electrode localizations have been debated, leading to some uncertainty regarding these first clinical results (Yelnik, 2007). Three years later, two double-blind controlled studies in six PD patients concluded that freezing of gait can be mildly improved by PPN stimulation but the overall results remain disappointing (Ferraye et al., 2010; Moro et al., 2010). These heterogeneous outcomes emphasize the need to determine the optimal surgical target within the MLR. It may be the case that treating PD patients suffering from failure of gait initiation versus falling may require specifically targeting the $\mathrm{CN}$ and the dorsal part of the PPN, respectively.

Using the IOM task, we also identified brain areas that are important for maintaining and using spatial information. At the cortical level, the comparison between brain activity associated with IOM and IG conditions highlighted a network that included two specific regions: the lateral intraparietal (LIP) area within the posterior parietal cortex and the dorsolateral prefrontal cortex (DLPFC). It is well established from studies in monkeys and humans that the posterior parietal cortex, and more specifically LIP, is crucial for spatial attention (Corbetta et al., 2002; Bisley and Goldberg, 2003; Owen et al., 2006). Attending to and tracking an object in space also involves working memory (Ricciardi et al., 2006; Offen et al., 2010), which may explain the DLPFC activation in our IOM task.

At the brainstem level, we found an activation in the MLR associated with fast IOM versus normal IOM. This activation is located more ventrally and more anteriorly to the one corresponding to fast IG versus normal IG. Anatomically, this cluster of activation includes the ventral part of the PPN and extends ventrally into the medial reticular formation and includes also a small lateral part of the substantia nigra pars reticulata. This specific area of the mesencephalus may be involved in integrating salient environmental information during gait. Interestingly, one portion of this ventral part of the PPN is active during both motor and nonmotor imagery. This may be explained by the fact that controlling gait requires continuous adjustments to spatial cues to smoothly adapt locomotion to changing environmental conditions. This highly integrative region in the MLR might correspond to the pars compacta of the PPN that is richer in cholinergic neurons and known to be involved in both motor and nonmotor functions, as already demonstrated in animals (Kobayashi and Isa, 2002; Winn, 2008; Karachi et al., 2010) and in humans (Rinne et al., 2008; Stefani et al., 2010). Moreover, cholinergic neurons have been shown to degenerate in progressive supranuclear palsy and PD (Hirsch et al., 1987), especially in patients with axial symptoms (Karachi et al., 2010), and it is well known that these patients also develop cognitive impairments (Vandenbossche et al., 2011). We suggest that one of the pathophysiological mechanisms involved in gait and cognitive dysfunctions could involve these cholinergic PPN neurons.

\section{References}

Alam M, Schwabe K, Krauss JK (2011) The pedunculopontine nucleus area: critical evaluation of interspecies differences relevant for its use as a target for deep brain stimulation. Brain 134:11-23.

Alderson HL, Latimer MP, Winn P (2008) A functional dissociation of the anterior and posterior pedunculopontine tegmental nucleus: excitotoxic lesions have differential effects on locomotion and the response to nicotine. Brain Struct Funct 213:247-253.

Aravamuthan BR, Muthusamy KA, Stein JF, Aziz TZ, Johansen-Berg H (2007) Topography of cortical and subcortical connections of the human pedunculopontine and subthalamic nuclei. Neuroimage 37:694-705.

Aravamuthan BR, McNab JA, Miller KL, Rushworth M, Jenkinson N, Stein JF, Aziz TZ (2009) Cortical and subcortical connections within the pedunculopontine nucleus of the primate Macaca mulatta determined using probabilistic diffusion tractography. J Clin Neurosci 16:413-420.

Bakker M, De Lange FP, Helmich RC, Scheeringa R, Bloem BR, Toni I (2008) Cerebral correlates of motor imagery of normal and precision gait. Neuroimage 41:998-1010. 
Bardinet E, Bhattacharjee M, Dormont D, Pidoux B, Malandain G, Schüpbach M, Ayache N, Cornu P, Agid Y, Yelnik J (2009) A threedimensional histological atlas of the human basal ganglia. II. Atlas deformation strategy and evaluation in deep brain stimulation for Parkinson disease. J Neurosurg 110:208-219.

Bisley JW, Goldberg ME (2003) Neuronal activity in the lateral intraparietal area and spatial attention. Science 299:81-86.

Bohnen NI, Müller ML, Koeppe RA, Studenski SA, Kilbourn MA, Frey KA, Albin RL (2009) History of falls in Parkinson disease is associated with reduced cholinergic activity. Neurology 73:1670-1676.

Corbetta M, Kincade JM, Shulman GL (2002) Neural systems for visual orienting and their relationships to spatial working memory. J Cogn Neurosci 14:508-523.

Ferraye MU, Debû B, Fraix V, Goetz L, Ardouin C, Yelnik J, Henry-Lagrange C, Seigneuret E, Piallat B, Krack P, Le Bas JF, Benabid AL, Chabardès S, Pollak P (2010) Effects of pedunculopontine nucleus area stimulation on gait disorders in Parkinson's disease. Brain 133:205-214.

Fink GR, Frackowiak RS, Pietrzyk U, Passingham RE (1997) Multiple nonprimary motor areas in the human cortex. J Neurophysiol 77:2164-2174.

Friston KJ, Fletcher P, Josephs O, Holmes A, Rugg MD, Turner R (1998) Event-related fMRI: characterizing differential responses. Neuroimage $7: 30-40$.

Grillner S (1981) Control of locomotion in bipeds, tetrapods and fish. In: Handbook of physiology: the nervous system II (Brooks VB, ed.), pp 1199-1236. Baltimore: American Physiological Society.

Haaland KY, Harrington DL, Knight RT (2000) Neural representations of skilled movement. Brain 123:2306-2313.

Hirsch EC, Graybiel AM, Duyckaerts C, Javoy-Agid F (1987) Neuronal loss in the pedunculopontine tegmental nucleus in Parkinson disease and in progressive supranuclear palsy. Proc Natl Acad Sci U S A 84:5976-5980.

Iseki K, Hanakawa T, Shinozaki J, Nankaku M, Fukuyama H (2008) Neural mechanisms involved in mental imagery and observation of gait. Neuroimage 41:1021-1031.

Jahn K, Deutschländer A, Stephan T, Strupp M, Wiesmann M, Brandt T (2004) Brain activation patterns during imagined stance and locomotion in functional magnetic resonance imaging. Neuroimage 22:1722-1731.

Jahn K, Deutschländer A, Stephan T, Kalla R, Wiesmann M, Strupp M, Brandt T (2008) Imaging human supraspinal locomotor centers in brainstem and cerebellum. Neuroimage 39:786-792.

Janssen L, Meulenbroek RG, Steenbergen B (2011) Behavioral evidence for left-hemisphere specialization of motor planning. Exp Brain Res 209:65-72.

Jeannerod M, Decety J (1995) Mental motor imagery: a window into the representational stages of action. Curr Opin Neurobiol 5:727-732.

Jenkinson N, Nandi D, Muthusamy K, Ray NJ, Gregory R, Stein JF, Aziz TZ (2009) Anatomy, physiology, and pathophysiology of the pedunculopontine nucleus. Mov Disord 24:319-328.

Jordan LM, Liu J, Hedlund PB, Akay T, Pearson KG (2008) Descending command systems for the initiation of locomotion in mammals. Brain Res Rev 57:183-191.

Karachi C, Grabli D, Bernard FA, Tandé D, Wattiez N, Belaid H, Bardinet E, Prigent A, Nothacker HP, Hunot S, Hartmann A, Lehéricy S, Hirsch EC, François C (2010) Cholinergic mesencephalic neurons are involved in gait and postural disorders in Parkinson disease. J Clin Invest 120:2745-2754.

Kobayashi Y, Isa T (2002) Sensory-motor gating and cognitive control by the brainstem cholinergic system. Neural Netw 15:731-741.

la Fougère $\mathrm{C}$, Zwergal A, Rominger A, Förster S, Fesl G, Dieterich M, Brandt T, Strupp M, Bartenstein P, Jahn K (2010) Real versus imagined locomotion: a [18F]-FDG PET-fMRI comparison. Neuroimage 50:1589-1598.

Mazzone P, Lozano A, Stanzione P, Galati S, Scarnati E, Peppe A, Stefani A (2005) Implantation of human pedunculopontine nucleus: a safe and clinically relevant target in Parkinson's disease. Neuroreport 16:1877-1881.

Mazzone P, Sposato S, Insola A, Dilazzaro V, Scarnati E (2008) Stereotactic surgery of nucleus tegmenti pedunculopontine [corrected]. Br J Neurosurg 22 [Suppl 1]:S33-S40.

Mazzone P, Scarnati E, Garcia-Rill E (2011) Commentary: the pedunculopontine nucleus: clinical experience, basic questions and future directions. J Neural Transm 118:1391-1396.
Mesulam MM, Geula C, Bothwell MA, Hersh LB (1989) Human reticular formation: cholinergic neurons of the pedunculopontine and laterodorsal tegmental nuclei and some cytochemical comparisons to forebrain cholinergic neurons. J Comp Neurol 283:611-633.

Mori S, Sakamoto T, Ohta Y, Takakusaki K, Matsuyama K (1989) Sitespecific postural and locomotor changes evoked in awake, freely moving intact cats by stimulating the brainstem. Brain Res 505:66-74.

Mori S, Matsui T, Mori F, Nakajima K, Matsuyama K (2000) Instigation and control of treadmill locomotion in high decerebrate cats by stimulation of the hook bundle of Russell in the cerebellum. Can J Physiol Pharmacol 78:945-957.

Moro E, Hamani C, Poon YY, Al-Khairallah T, Dostrovsky JO, Hutchison WD, Lozano AM (2010) Unilateral pedunculopontine stimulation improves falls in Parkinson's disease. Brain 133:215-224.

Muthusamy KA, Aravamuthan BR, Kringelbach ML, Jenkinson N, Voets NL, Johansen-Berg H, Stein JF, Aziz TZ (2007) Connectivity of the human pedunculopontine nucleus region and diffusion tensor imaging in surgical targeting. J Neurosurg 107:814-820.

Offen S, Gardner JL, Schluppeck D, Heeger DJ (2010) Differential roles for frontal eye fields (FEFs) and intraparietal sulcus (IPS) in visual working memory and visual attention. J Vis 10:28.

Oldfield RC (1971) The assessment and analysis of handedness: the Edinburgh inventory. Neuropsychologia 9:97-113.

Olszewski J, Baxter D (1954) Cytoarchitecture of the human brainstem. Philadelphia: J. B. Lippincott.

Owen AM, Coleman MR, Boly M, Davis MH, Laureys S, Pickard JD (2006) Detecting awareness in the vegetative state. Science 313:1402.

Penfield W (1954) Mechanisms of voluntary movement. Brain 77:1-17.

Piallat B, Chabardès S, Torres N, Fraix V, Goetz L, Seigneuret E, Bardinet E, Ferraye M, Debu B, Krack P, Yelnik J, Pollak P, Benabid AL (2009) Gait is associated with an increase in tonic firing of the sub-cuneiform nucleus neurons. Neuroscience 158:1201-1205.

Ricciardi E, Bonino D, Gentili C, Sani L, Pietrini P, Vecchi T (2006) Neural correlates of spatial working memory in humans: a functional magnetic resonance imaging study comparing visual and tactile processes. Neuroscience 139:339-349.

Rinne JO, Ma SY, Lee MS, Collan Y, Röyttä M (2008) Loss of cholinergic neurons in the pedunculopontine nucleus in Parkinson's disease is related to disability of the patients. Parkinsonism Relat Disord 14:553-557.

Shik ML, Severin FV, Orlovskii GN (1966) [Control of walking and running by means of electric stimulation of the midbrain]. Biofizika 11:659-666.

Snijders AH, Leunissen I, Bakker M, Overeem S, Helmich RC, Bloem BR, Toni I (2011) Gait-related cerebral alterations in patients with Parkinson's disease with freezing of gait. Brain 134:59-72.

Stefani A, Pierantozzi M, Ceravolo R, Brusa L, Galati S, Stanzione P (2010) Deep brain stimulation of pedunculopontine tegmental nucleus (PPTg) promotes cognitive and metabolic changes: a target-specific effect or response to a low-frequency pattern of stimulation? Clin EEG Neurosci 41:82-86.

Takakusaki K (2008) Forebrain control of locomotor behaviors. Brain Res Rev 57:192-198.

Vandenbossche J, Deroost N, Soetens E, Spildooren J, Vercruysse S, Nieuwboer A, Kerckhofs E (2011) Freezing of gait in Parkinson disease is associated with impaired conflict resolution. Neurorehabil Neural Repair 25:765-773.

Wang J, Wai Y, Weng Y, Ng K, Huang YZ, Ying L, Liu H, Wang C (2009) Functional MRI in the assessment of cortical activation during gaitrelated imaginary tasks. J Neural Transm 116:1087-1092.

Winn P (2008) Experimental studies of pedunculopontine functions: are they motor, sensory or integrative? Parkinsonism Relat Disord 14 [Suppl 2]:S194-S198.

Yelnik J (2007) PPN or PPD, what is the target for deep brain stimulation in Parkinson's disease? Brain 130:e79; author reply e80.

Yelnik J, Bardinet E, Dormont D, Malandain G, Ourselin S, Tandé D, Karachi C, Ayache N, Cornu P, Agid Y (2007) A three-dimensional, histological and deformable atlas of the human basal ganglia. I. Atlas construction based on immunohistochemical and MRI data. Neuroimage 34:618-638. 